Research Paper

\title{
Salmonella Overcomes Drug Resistance in Tumor through P-glycoprotein Downregulation
}

\author{
Chih-Jen Yang1, 2, 3, Wen-Wei Chang4, 5, Song-Tao Lin'6, Man-Chin Chen ${ }^{6}$, Che-Hsin Lee, $7 \bowtie$ \\ 1. Department of Internal Medicine, Kaohsiung Municipal Ta-Tung Hospital, Kaohsiung Medical University, Kaohsiung, Taiwan; \\ 2. Division of Pulmonary and Critical Care Medicine, Department of Internal Medicine, Kaohsiung Medical University Hospital, Kaohsiung Medical \\ University, Kaohsiung, Taiwan; \\ 3. Faculty of Medicine, Department of Respiratory Therapy, College of Medicine, Kaohsiung Medical University, Taiwan; \\ 4. Department of Biomedical Sciences, College of Medical Science and Technology, Chung Shan Medical University, Taichung, Taiwan; \\ 5. Department of Medical Research, Chung Shan Medical University Hospital, Taichung, Taiwan; \\ 6. Department of Biological Sciences, National Sun Yat-sen University, Kaohsiung, Taiwan; \\ 7. Department of Medical Research, China Medical University Hospital, China Medical University, Taichung 404, Taiwan
}

$\triangle$ Corresponding author: Dr. Che-Hsin Lee, Department of Biological Sciences, National Sun Yat-sen University, Kaohsiung, Taiwan, 70 Lienhai Rd. Kaohsiung 80424, Taiwan. E-mail: chlee@mail.nsysu.edu.tw

(C) Ivyspring International Publisher. This is an open access article distributed under the terms of the Creative Commons Attribution (CC BY-NC) license (https://creativecommons.org/licenses/by-nc/4.0/). See http://ivyspring.com/terms for full terms and conditions.

Received: 2017.10.11; Accepted: 2018.02.14; Published: 2018.03.09

\begin{abstract}
Chemotherapy is one of effective methods for the treatment of tumor. Patients often develop drug resistance after chemotherapic cycles. Salmonella has potential as antitumor agent. Salmonella used in tandem with chemotherapy had additive effects, providing a rationale for using tumor-targeting Salmonella in combination with conventional chemotherapy. To improve the efficacy and safety of Salmonella, a further understanding of Salmonella interactions with the tumor microenvironment is required. The presence of plasma membrane multidrug resistance protein P-glycoprotein (P-gP) is highly relevant for the success of chemotherapy. Following Salmonella infection, dose-dependent downregulation of P-gP expressions were examined. Salmonella significantly decreased the efflux capabilities of P-gp, as based on the influx of Rhodamine 123 assay. In addition, Salmonella significant reduced the protein express the expression levels of phosph-protein kinase B (P-AKT), phosph-mammalian targets of rapamycin (P-mTOR), and phosph-p70 ribosomal s6 kinase (P-p70s6K) in tumor cells. The Salmonella-induced downregulation of $\mathrm{P}$-gP was rescued by transfection of cells with active P-AKT. Our results demonstrate that Salmonella in tumor sites leads to decrease the expression of P-gP and enhances the combination of Salmonella and 5-Fluorouracil therapeutic effects.
\end{abstract}

Key words: Salmonella, P-glycoprotein, 5-Fluorouracil, tumor, combination therapy

\section{Introduction}

The proliferation of drug resistant tumor cells is believed to relapse in most patients after several cycles of chemotherapy. The growing evidences point out that the tumor microenvironment plays an important role in drug resistance [1]. P-glycoprotein 1 (P-gp) is the multidrug resistance protein 1 (MDR1) or ATP-binding cassette, sub-family $\mathrm{B}$ member 1 (ABCB1). The P-gp is an important protein of the cell membrane that pumps chemotherapeutic drugs out of cells [2]. Many tumors have higher levels of P-gp that correlates with drug resistance [3].

Salmonella targets to tumor sites and arrests tumor growth $[4,5]$. The combination of Salmonella and chemotherapy acted additively to delay tumor growth and prolong the survival time of tumorbearing mice [5- 7]. Previously, we have shown that Salmonella increases the efficiency of cisplatin via enhancement of gap junction [8]. Meanwhile, Salmonella can target to tumors and modulate numerous signaling pathways of tumor cells [9]. We hypothesize that Salmonella not only enhances drug diffusion via upregulation gap junction, but also decreases drug efflux through downregulation P-gp. This study provides evidence for the mechanism of the regulation of P-gp by Salmonella and validates the incorporation of Salmonella as a novel therapeutic 
strategy against tumor.

\section{Material and Methods}

\section{Bacteria, cell lines, plasmid, reagents and animals}

Salmonella enterica serovar choleraesuis (S. Choleraesuis; S.C.) (ATCC 15480) was obtained from Bioresources Collection and Research Center (Hsinchu, Taiwan) [10]. B16F10 (mouse melanoma) [11] and 4T1 (mouse breast cancer) [12] cells were maintained in culture dish with Dulbecco's Modified Eagle Medium containing $1 \%$ antibiotics (100 units/mL penicillin and $100 \mu \mathrm{g} / \mathrm{mL}$ streptomycin), $2 \mathrm{mM}$ l-glutamine and $10 \%$ fetal bovine serum. Rhodamine 123 (Rho-123) and 5-Fluorouracil (5-FU) were purchased from Sigma Aldrich. Constitutively active AKT plasmid was kindly provided by Dr. Chiau-Yuang Tsai (Department of molecular immunology, Osaka University) [13]. Cells were treated with plasmids for $16 \mathrm{~h}$ in serum free medium prior to adding Salmonella (multiplicity of infection (MOI) = 10) into cells for $90 \mathrm{~min}$. C57BL/6 (B16F10 mouse tumor model) and BALB/c (4T1 mouse tumor model) female mice were purchased from the National Laboratory Animal Center of Taiwan. The animals were maintained in a pathogen-free animal care facility in isothermal conditions with regular photoperiods. The experimental protocol adhered to the rules of the Animal Protection Act of Taiwan and was approved by the Laboratory Animal Care and Use Committee of the National Sun Yat-sen University.

\section{Western Blotting Analysis}

Bicinchoninic acid (BCA) protein assay (Pierce Biotechnology, Rockford, IL, USA) was used to determine the protein content in each sample. Quantified each sample concentration to $60-80 \mu \mathrm{g}$ and proteins were fractionated on SDS-PAGE, transferred onto Hybond enhanced chemiluminescence nitrocellulose membranes (Amersham, Little Chalfont, UK) and detected with antibodies against P-gp (GeneTex, Inc. Irvine, CA, USA), the mammalian target of rapamycin (mTOR) (Cell Signaling, Danvers, MA, USA), phosph-mTOR (Cell Signaling), protein kinase B (AKT) (Santa Cruz Biotechnology, Inc. Santa Cruz, CA, USA), phosph-AKT (Santa Cruz Biotechnology, Inc.), p70s6K (Cell Signaling), phosph-p70s6K (Cell Signaling), caspase 3 (Cell Signaling), $\beta$-actin (Sigma Aldrich). Rabbit anti-mouse IgG-peroxidase antibody (Sigma Aldrich) and goat anti-rabbit IgG-peroxidase antibody (Sigma Aldrich) were used as the secondary antibody and protein-antibody complexes were visualized by enhanced chemiluminescence system. The Western blotting signals were quantified with
ImageJ software (rsbweb.nih.gov/ij/) [14, 15].

\section{P-gp functional assay}

B16F10 or 4T1 cells were plated in 12 well culture plates for $24 \mathrm{~h}$, and treated with various MOI of Salmonella for $4 \mathrm{~h}$. The cells were washed with PBS and added the antibiotic medium. Retention of Rho-123 was measured to evaluate P-gp functional activity in B16F10 and 4T1 cells. Intracellular Rho-123 was determined from the fluorescence (Ex. 530nm Em. $590 \mathrm{~nm}$ ) by spectrometer (BMG Labtech, Headquarters Germany). The accumulation of Rho-123 in cells was also analyzed using a fluorescence microscope. Cell proliferation was assessed by the colorimetric WST-8 assay (Dojindo Labs, Tokyo, Japan) according to the manufacturer's instructions [16].

\section{Animal study}

Mice were inoculated subcutaneously (s.c.) with $10^{6} \mathrm{~B} 16 \mathrm{~F} 10$ or $4 \mathrm{~T} 1$ cells at day 0 , and at day 7 nodules developed at all injection sites with approximately tumor volume of $80 \mathrm{~mm}^{3}$. Groups of tumor-bearing mice were injected intraperitoneally (i.p.) with Salmonella $2 \times 10^{6}$ colony-forming units (cfu) at day 7 followed by 5 -FU $(40 \mathrm{mg} / \mathrm{kg})$ treatment on days 9,11 , and 13 , or with either treatment alone. All of the mice were monitored for tumor growth as previously described [8].

\section{Statistical analysis}

The ANOVA was used to determine differences between groups. Any $P$ value less than 0.05 is regarded statistically significant.

\section{Results}

\section{Intracellular accumulation of Rho-123 after Salmonella infection}

To analysis whether Salmonella could regulate P-gp transport activity after Salmonella infection in tumor cells, the intracellular accumulation of Rho-123 was assessed. The P-gp functionality was measured by detecting the intracellular accumulation of fluorescent (Rho-123). As shown in Fig. 1A, Rho-123 was detected modestly at the nuclear periphery and in the cytoplasm in control cells, whereas it was detected exclusively in the cytoplasm in the cells treated with Salmonella. We also observed the similar phenomenon in B16F10 cells (Fig. 1A). By measuring the fluorescence values, the accumulation of Rho-123 was significantly more in the Salmonella-treated cells than that in the control cells (Fig. 1B). These results show that Salmonella increases the accumulation of Rho-123, indicating that Salmonella suppresses P-gp transport activity. 
A

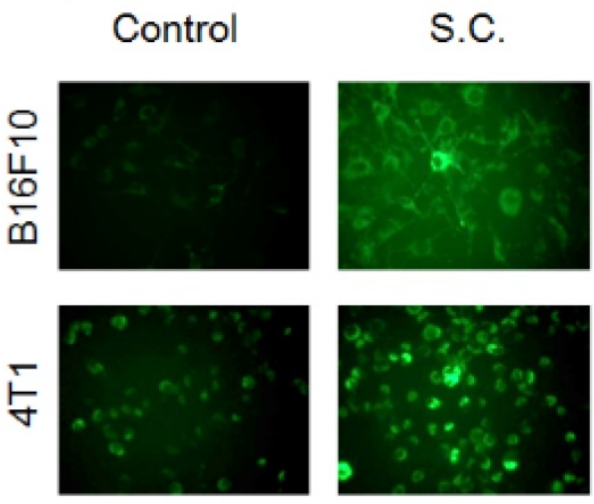

B

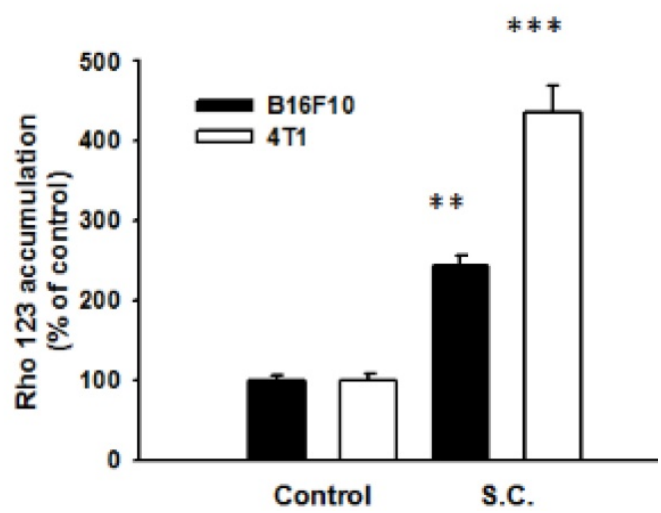

Figure 1. Rhodamine 123 (Rho-123) accumulation in B 16 F 10 and 4T1 cells treated with Salmonella (S.C.). B16F10 and 4T1 cells were infected with S.C. for $4 \mathrm{~h}$, washed and incubated with culture medium for $2 \mathrm{~h}$ prior to addition of Rho-123. (A) The accumulation of Rho-123 in cells was analyzed using a fluorescence microscope. (B) The Rho-123 were determined from the fluorescence by using spectrofluorometer. $* * P<0.01 ; * * * P<0.001$.

A

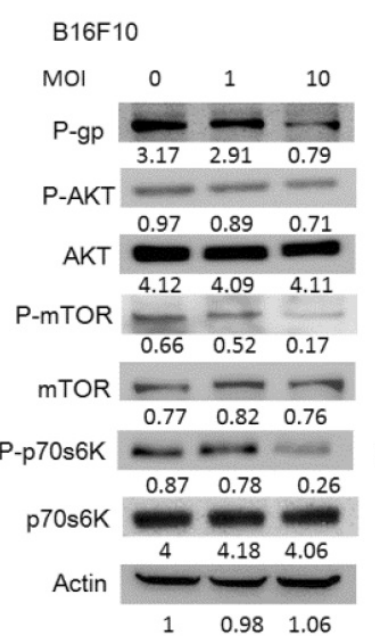

B

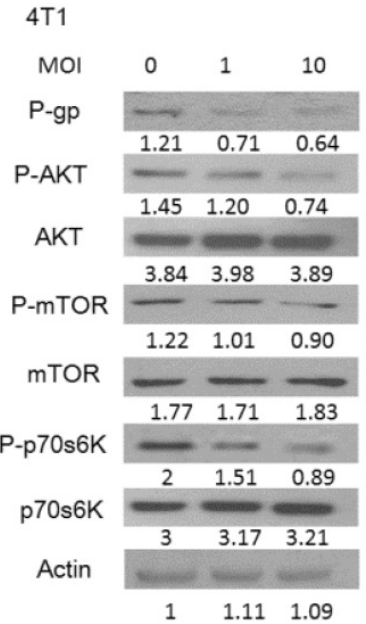

Figure 2. Salmonella (S.C.) regulated the expression of P-glycoprotein (P-gp), and AKT/mTOR signaling pathway. The expression of P-gp, AKT, mTOR, p70s6K was measured in B16F10 (A) and 4T1 (B) cells by Western blotting analysis. The $\beta$-actin expression served as loading controls for and total protein. Each experiment was repeated three times with similar results.

\section{Salmonella reduced $\mathrm{P}$-gp expression through P-AKT/ P-mTOR pathway}

To gain insights into the role of P-gp on Salmonella infection, we used Western blotting analysis to study the correlation between P-gp and Salmonella infection. Fig. 2 shows that the protein levels of P-gp were decreased in Salmonella-treated tumor cells. Furthermore, the AKT / mTOR signaling pathway can promote $\mathrm{P}$-gp protein synthesis through phosphorylation of p70s6K $[17,18]$. In dose dependent manner, Salmonella significantly reduced the phosphorylation of AKT, mTOR, and p70s6K in B16F10 and 4T1 cells (Fig. 2). These results suggest that reduction of P-gp expression in tumor cells was

associated with inhibition AKT/mTOR/p70s6K signaling pathway during Salmonella infection.

\section{Salmonella increased susceptibility of tumor cells to 5-FU}

Furthermore, we examined the downregulation of P-gp expression by Salmonella infection upon the sensitivity of the tumor cells to killing by 5-FU that induce cell death. Herein, the proliferation of tumor cell was measured after 5-FU, Salmonella or Salmonella combined with 5-FU treatment. The results show that the amounts of tumor cells were lowest when tumor cells were treated with Salmonella combined 5-FU (40 $\mu \mathrm{M})$ compared with the control group (Fig. 3A and C). Treatment with 5-FU significantly enhanced cell death in Salmonella-infected cells as compared to uninfected cells. Meanwhile, we also observed that Salmonella and 5-FU increased the apoptosis marker (cleaved caspase 3) (Fig. 3B and D). Collectively, these results indicate that Salmonella may inhibit the proliferation of tumor cells through the enhancing susceptibility of tumor cells to 5 -FU.

\section{Salmonella suppressed P-gp expression via an inhibition AKT signaling pathway}

Consequently, we next examined the extent to which changes in P-gp expression contributed to the Salmonella-inhibited AKT/mTOR/p70s6K signaling pathway. By transfecting constitutively active AKT plasmid, the AKT/mTOR/p70s6K signaling pathway can be reversed [16]. Salmonella did not inhibit the constitutively active AKT. As shown in Fig. 4, Salmonella lost the ability to suppress the expression of the P-gp through downregulation AKT/mTOR/ p70s6K signaling pathway after transfecting constitutively active AKT plasmid. The similar results were also observed in the expression of cleaved-caspase 3 in the B16F10 and 4T1 cells treated 
with 5-FU in combination with Salmonella. After transfecting constitutively active AKT plasmid in two tumor cells, the expression of cleaved-caspase 3 was reduced after the combo therapy (Fig. 5). The results demonstrate that downregulation $\mathrm{AKT} / \mathrm{mTOR}$ signaling pathway is necessary for Salmonella-reduced P-gp expression in tumor cells.
A

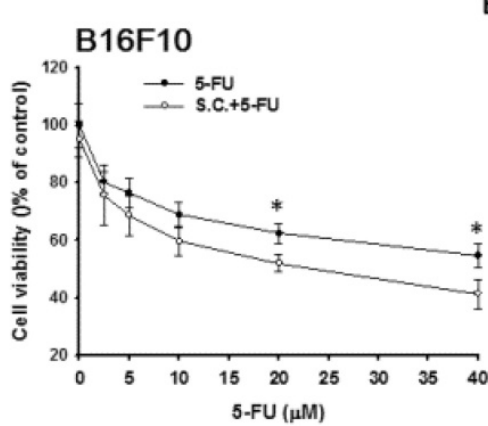

C

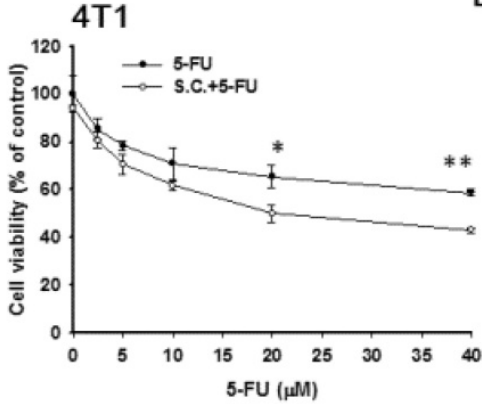

\section{B16F10}

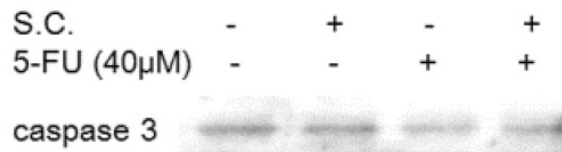

Cleaved-caspase 3

Actin

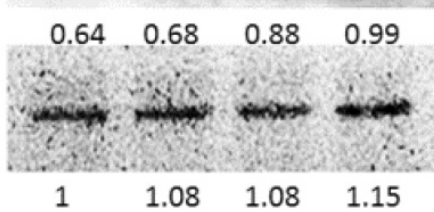

4T1

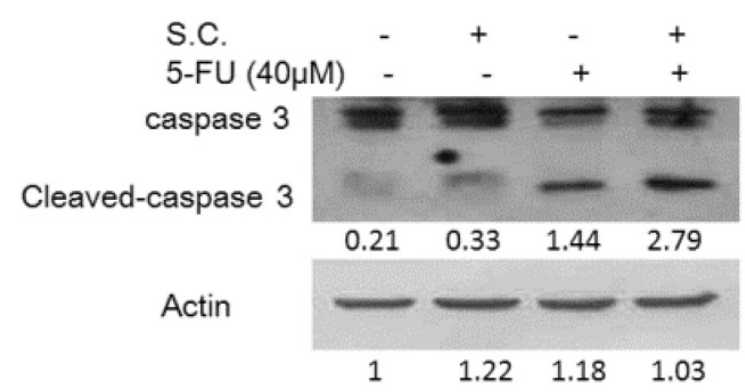

Figure 3. Salmonella (S.C.)-regulated P-glycoprotein (P-gP) expression in conjunction with 5-FU exerted cytotoxic effects on B 16 F 10 and 4 T 1 cells. Salmonella-treated $(\mathrm{MOI}=10)$, or control cells were exposed to 5 -FU $(0-40 \mu \mathrm{M})$ for $48 \mathrm{~h}$ followed by determination of their viability by the WST-8 assay in B16F10 (A) and 4T1 (C) cells. Data are expressed as mean \pm SD of hexaplicate determinations. Salmonella-treated (MOI=10), or control cells were exposed to 5-FU $(40 \mu \mathrm{M})$ for $48 \mathrm{~h}$ followed by determination of caspase 3 expression by the Western blotting analysis in B16F10 (B) and 4T1 (D) cells. Multiplicity of infection (MOI). $* P<0.05$.

A

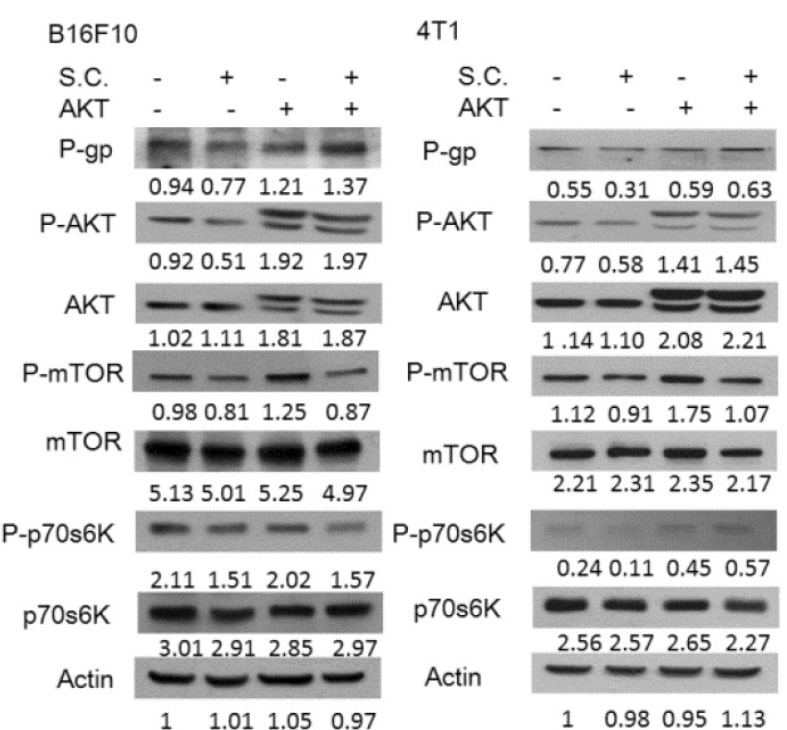

Figure 4. Constitutively active-AKT reduced the effects of Salmonella (S.C.). The B16F10 and 4T1 cells were transfected with constitutively active AKT plasmid $(5 \mu \mathrm{g})$ for $16 \mathrm{~h}$ prior to treatment with Salmonella $(\mathrm{MOI}=10)$ or not for $4 \mathrm{~h}$. The expression of P-glycoprotein (P-gp), AKT, mTOR, p70s6K protein in B16F10 (A) and 4Tl cells (B) was determined. The $\beta$-actin expression served as loading controls for and total protein. Each experiment was repeated three times with similar results.
B16F10

$\begin{array}{lllllllll}\text { S.C. } & - & + & - & + & - & + & - & + \\ \text { 5-FU }(40 \mu \mathrm{M}) & - & - & + & + & - & - & + & + \\ \text { AKT } & - & - & - & - & + & + & + & +\end{array}$

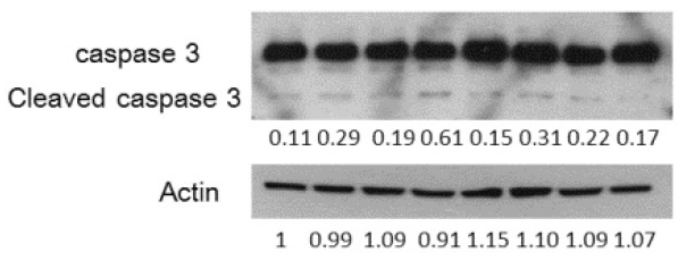

B

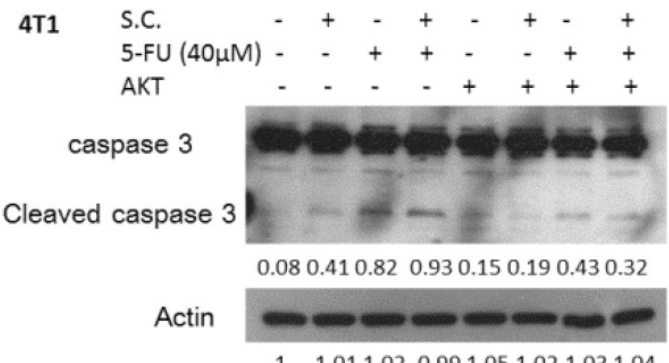

Figure 5. Constitutively active-AKT decreased the effects of Salmonella (S.C.) in combination with 5-FU. The B16F10 and 4T1 cells were transfected with constitutively active AKT plasmid $(5 \mu \mathrm{g})$ for $16 \mathrm{~h}$ prior to treatment with Salmonella $(\mathrm{MOI}=10)$ or not for $4 \mathrm{~h}$. Salmonella-treated (MOI=10), or control cells were exposed to 5 -FU $(40 \mu \mathrm{M})$ for $48 \mathrm{~h}$ followed by determination of caspase 3 expression by the Western blot analysis in B16F10 (A) and 4T1 (B) cells. Multiplicity of infection (MOI). 
A

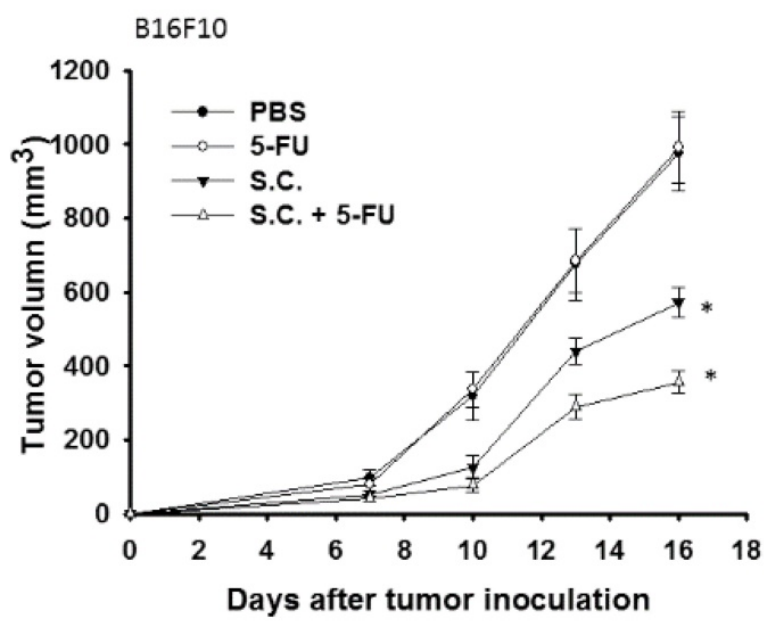

B

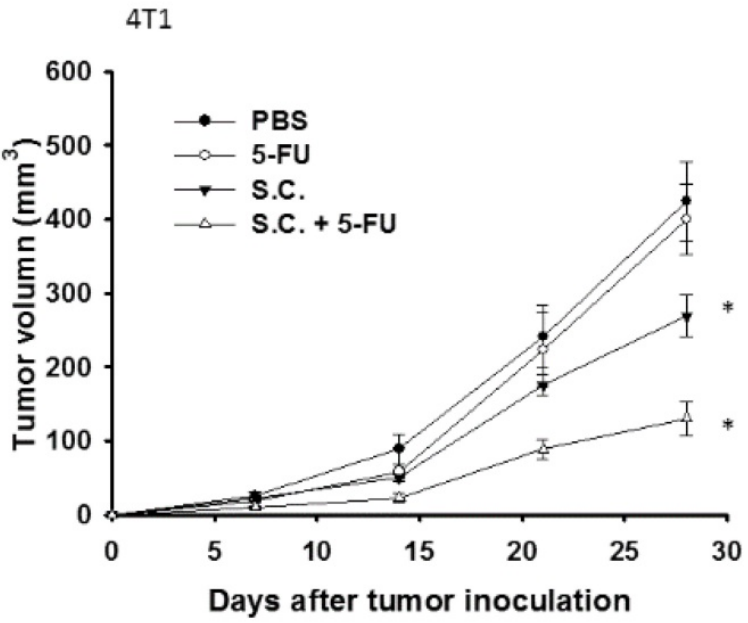

Figure 6. Additive antitumor effects of Salmonella (S.C.) in combination with 5-FU on subcutaneous B 16 F 10 and 4T1 tumors. Groups of 8 mice that had been inoculated s.c. with B16F10 (a) and 4Tlcells (b) at day 0 were treated i.p. with Salmonella $\left(2 \times 10^{6} \mathrm{cfu}\right)$ at day 7 followed by $5-\mathrm{FU}(40 \mathrm{mg} / \mathrm{kg})$ treatment at days 9,11 , and 13 , or with either treatment alone. Vehicle control mice received PBS. Tumor volumes (mean $\pm S E M, n=8$ ) among different treatment groups were compared in mice bearing tumors. $* P<0.05$.

\section{Salmonella in combination with 5-FU enhanced the antitumor activity}

The tumor growth of the mice bearing B16F10 or 4T1 tumors was evaluated the antitumor effects of Salmonella. As shown in Fig. 6 A and B, Salmonella significantly inhibited tumor growth in comparison with that in PBS-treated control mice. The low-dose 5-FU did not show significant antitumor activity. As expected, the combination therapy of Salmonella and 5-FU enhanced the antitumor activity. In B16F10 and 4T1 tumor model, the tumor volume of mice treated with the combination therapy reduced by $37.7 \%$ and $51.67 \%$ compared with those in the Salmonella-treated group, respectively. Taken together, Salmonella alone could reduce tumor growth in murine tumor models. Furthermore, the combination therapy had the additive antitumor effects.

\section{Discussion}

The effects of the combinational treatment of 5-FU and Salmonella in vitro and in vivo were determined by using mouse melanoma and breast tumor models. The combination of Salmonella and 5-FU inhibits tumor growth and tumor size compared to 5-FU alone, suggesting that Salmonella can increase the efficacy of 5-FU. However, the detailed mechanism of the combinational therapy remained uncertain. In this study, we used mouse tumor models to determine the mechanisms of Salmonellainduced signaling and the consequences of the combination therapy. Salmonella was demonstrated the ability to decrease the expression of P-gp through posttranscriptionally regulated [19]. Some studies also demonstrated downregulation of $\mathrm{p}$-gp expression via inhibition of AKT/mTOR pathway [17]. There is a growing evidence that an increase in chemotherapymediated response to P-gp downregulation [20]. There is extensive literature of tumor-targeting Salmonella increasing the efficacy of chemotherapy [21-24]. Salmonella recruited peripheral immune cells to the tumor by upregulating interferon $\gamma$ and $\mathrm{T}$ cells are also involved in the modulation of Salmonella-induced host immunity [25, 26]. Thus, some studies may suggest that tumor necrosis factor a is response for main cells infiltrating Salmonellatreated tumors [27]. Salmonella has been used as an antitumor agent that specifically proliferates within tumors and inhibits their growth [28]. In addition, Salmonella cannot disperse throughout the tumor and this is the disadvantage for its use as an anticancer agent [28]. Our previous studies suggested that Salmonella disperses within tumors and inhibits tumor growth when combined with chemotherapy $[7,8]$. There is a significant increase of apoptosis in 5-FU-treated cell. Cell proliferation analysis and Western blotting assay showed a significant increase in cell death of Salmonella combined with 5-FU treated tumors compared to control. Downregulation of P-gp may allow high intracellular cytotoxic drug concentrations in tumor cells. The P-gp expression in tumor cells may influence the clinical response to chemotherapy. To show the results, Salmonella manipulate P-gp in conjunction with 5-FU treatment may provide new insights for tumor therapy. The $5-\mathrm{FU}$ is one of the most commonly used drugs to treat cancer including breast, colon and some skin cancers. Therefore, the major limiting factor in the use of 5-FU 
is the side effects in normal tissue including diarrhea, month ulcers, and hair thinning. This would allow the use of lower 5-FU concentration, thus decreasing the side effects. Salmonella provides to accelerate cell death in tumor cells while decreasing P-gp expression. Recent studies revealed that resveratrol decreases in P-gp expression is regulated by AKT/mTOR signaling pathways [29]. Previously, we demonstrated that AKT/mTOR signaling is involved in the cell-defense elimination of Salmonella. The AKT/mTOR signaling pathway negatively regulates some protein syntheses that are critical for tumor cell survival $[11,16]$. We determined that the levels of phosphorylated AKT, mTOR, and p70s6K were significantly decreased in Salmonella-treated cancer cells. Herein, we investigated the effect of Salmonella on P-gp expression and function. We found that Salmonella-inhibited activation of AKT/mTOR pathway and are thought to play an important role in the downregulation of P-gp. By using of the cytoxicity effect of 5-FU and the pleiotropic activities of Salmonella, we suggest that Salmonella combining with 5-FU appears to aim at reversing cancer drug resistance.

\section{Abbreviations}

P-gp: P-glycoprotein; P-AKT: phosph-protein kinase B; P-mTOR: phosph-mammalian targets of rapamycin; P-p70s6K: phosph-p70 ribosomal s6 kinase; 5-FU: 5-Fluorouracil; Rho-123: Rhodamine 123: MDR1: multidrug resistance protein 1; ABCB1: ATP-binding cassette, sub-family B member 1; S.C.: Salmonella enterica serovar choleraesuis (S. Choleraesuis).

\section{Acknowledgements}

This work was supported by Ministry of Science and Technology, Taiwan (MOST 104-2320-B039-042MY3)

\section{Author Contributions}

C.J.Y. and C.H.L conceived and designed the experiments; C.J.Y, W.W.C, S.T.L. and M.C.C. performed the experiments; C.J.Y. and C.H.L analyzed the data; C.H.L contributed reagents/materials/ analysis tools; C.J.Y. and C.H.L wrote the paper.

\section{Competing Interests}

The authors have declared that no competing interest exists.

\section{References}

1. Yang CJ, Hung JY, Tsai MJ, et al. The salvage therapy in lung adenocarcinoma initially harbored susceptible EGFR mutation and acquired resistance occurred to the first-line gefitinib and second-line cytotoxic chemotherapy. BMC Pharmacol Toxicol. 2017; 18: 21.

2. Syed SB, Arya H, Fu IH, et al. Targeting P-glycoprotein: Investigation of piperine analogs for overcoming drug resistance in cancer. Sci Rep. 2017; 7: 7972 .
3. Su BH, Shieh GS, Tseng YL, et al. Etoposide enhances antitumor efficacy of MDR1-driven oncolytic adenovirus through autoupregulation of the MDR1 promoter activity. Oncotarget. 2015; 6: 38308-26.

4. Matsumoto Y, Miwa S, Zhang Y, et al. Intraperitoneal administration of tumor-targeting Salmonella typhimurium A1-R inhibits disseminated human ovarian cancer and extends survival in nude mice. Oncotarget. 2015; 6: 11369-77.

5. Zhang Y, Miwa S, Zhang N, et al. Tumor-targeting Salmonella typhimurium A1-R arrests growth of breast-cancer brain metastasis. Oncotarget. 2015; 6: 2615-22.

6. Hiroshima $\mathrm{Y}$, Zhang $\mathrm{Y}$, Murakami $\mathrm{T}$, et al. Efficacy of tumor-targeting Salmonella typhimurium A1-R in combination with anti-angiogenesis therapy on a pancreatic cancer patient-derived orthotopic xenograft (PDOX) and cell line mouse models. Oncotarget. 2014; 5: 12346-57.

7. Lee $\mathrm{CH}, \mathrm{Wu} \mathrm{CL}$, Tai YS, et al. Systemic administration of attenuated Salmonella choleraesuis in combination with cisplatin for cancer therapy. Mol Ther. 2015; 11: 707-16.

8. Chang WW, Lai CH, Chen MC, et al. Salmonella enhance chemosensitivity in tumor through connexin 43 upregulation. Int J Cancer. 2013; 133: 1926-35.

9. Lee $\mathrm{CH}$. Engineering bacteria toward tumor targeting for cancer treatment: current state and perspectives. Appl Microbiol Biotechnol. 2012, 93: 517-23.

10. Lee $\mathrm{CH}$, Nishikawa T, Kaneda Y. Salmonella mediated the hemagglutinating virus of Japan-envelope transfer suppresses tumor growth. Oncotarget. 2017; 8: 35048-60.

11. Tu DG, Chang WW, Lin ST, et al. Salmonella inhibits tumor angiogenesis by downregulation of vascular endothelial growth factor. Oncotarget. 2016; 7: 37513-23.

12. Chang WW, Kuan YD, Chen MC, et al. Tracking of mouse breast cancer stem-like cells with Salmonella. Exp Biol Med (Maywood). 2012; 237: 1189-96.

13. Shiau AL, Shen YT, Hsieh JL, et al. Scutellaria barbata inhibits angiogenesis through downregulation of HIF-1 a in lung tumor. Environ Toxicol. 2014; 29: 363-70.

14. Lin HC, Yang CJ, Kuan YD, et al. The inhibition of indoleamine 2, 3-dioxygenase 1 by connexin 43. Int J Med Sci. 2017; 14: 1181-8.

15. Tsao YT, Kuo CY, Kuan YD, et al. The extracts of Astragalus membranaceus inhibit melanogenesis through the ERK signaling pathway. Int J Med Sci. 2017;14: 1049-53.

16. Kuan $\mathrm{YD}$, Lee $\mathrm{CH}$. Salmonella overcomes tumor immune tolerance by inhibition of tumor indoleamine 2, 3-dioxygenase 1 expression. Oncotarget. 2016; 7: 374-85.

17. Chiu $\mathrm{LY}, \mathrm{Hu} \mathrm{ME}$, Yang TY, et al. Immunomodulatory protein from Ganoderma microsporum induces pro-death autophagy through Akt-mTOR-p70S6K pathway inhibition in multidrug resistant lung cancer cells. PLoS ONE. 2015; 10: e0125774.

18. Wang SF, Chou YC, Mazumder $\mathrm{N}$, et al. 7-Ketocholesterol induces P-glycoprotein through PI3K/mTOR signaling in hepatoma cells. Biochem Pharmacol. 2013; 86: 548-60.

19. Siccardi D, Mumy KL, Wall DM, et al. Salmonella enterica serovar Typhimurium modulates P-glycoprotein in the intestinal epithelium. Am J Physiol Gastrointest Liver Physiol. 2008; 294: G1392-400.

20. Mercado-Lubo R, Zhang Y, Zhao L, et al. A Salmonella nanoparticle mimic overcomes multidrug resistance in tumours. Nat Commun. 2016; 7: 12225.

21. Murakami T, DeLong J, Eilber FC, et al. Tumor-targeting Salmonella typhimurium A1-R in combination with doxorubicin eradicate soft tissue sarcoma in a patient-derived orthotopic xenograft (PDOX) model. Oncotarget. 2016; 7: 12783-90.

22. Yano S, Takehara K, Zhao M, et al. Tumor-specific cell-cycle decoy by Salmonella typhimurium A1-R combined with tumor-selective cell-cycle trap by methioninase overcome tumor intrinsic chemoresistance as visualized by FUCCI imaging. Cell Cycle. 2016; 15: 1715-23.

23. Kawaguchi K, Igarashi K, Murakami T, et al. Tumor-targeting Salmonella typhimurium A1-R combined with temozolomide regresses malignant melanoma with a BRAF-V600E mutation in a patient-derived orthotopic xenograft (PDOX) model. Oncotarget. 2016; 7: 85929-36.

24. Yamamoto $M$, Zhao M, Hiroshima $Y$, et al. Efficacy of tumor-targeting Salmonella A1-R on a melanoma patient-derived orthotopic Xenograft (PDOX) Nude-Mouse Model. PLoS One. 2016; 11: e0160882.

25. Lee $\mathrm{CH}, \mathrm{Wu} \mathrm{CL}$, Shiau AL. Toll-like receptor 4 mediates an antitumor host response induced by Salmonella choleraesuis. Clin Cancer Res. 2008; 14: 1905-12.

26. Lee $\mathrm{CH}, \mathrm{Hsieh} \mathrm{JL}, \mathrm{Wu} \mathrm{CL}$, et al. T cell augments the antitumor activity of tumor-targeting Salmonella. Appl Microbiol Biotechnol. 2011; 90: 1381-8.

27. Vendrell A, Gravisaco MJ, Pasetti MF, et al. A novel Salmonella Typhi-based immunotherapy promotes tumor killing via an antitumor Th1-type cellular immune response and neutrophil activation in a mouse model of breast cancer. Vaccine. 2011; 29: 728-36

28. Zheng $\mathrm{JH}$, Nguyen $\mathrm{VH}$, Jiang $\mathrm{SN}$, et al. Two-step enhanced cancer immunotherapy with engineered Salmonella typhimurium secreting heterologous flagellin. Sci Transl Med. 2017; 9: 376.

29. Wang $L$, Wang $C$, Jia $Y$, et al. Resveratrol increases anti-proliferative activity of bestatin through downregulating P-glycoprotein expression via inhibiting PI3K/Akt/mTOR pathway in K562/ADR cells. J Cell Biochem. 2016; 117:1233-9. 